enzymes. Therefore, the biotransformation of articaine occurs in both the liver and in plasma (resulting in low systemic toxicity).

Now that we have identified the most difficult teeth to anaesthetise and the potential advantages of articaine, one might ask is articaine effective as a supplementary buccal infiltration strategy in anaesthetising mandibular posterior teeth with irreversible pulpitis?

The authors of this recent randomised clinical trial (RCT) attempted to answer the above question. This RCT was carried out at Peking University in Beijing. The anaesthetic efficacy of $4 \%$ articaine, $2 \%$ lidocaine and $2 \%$ mepivacaine was investigated as supplemental buccal infiltration following unsuccessful lidocaine IANB. Each group comprised 52 patients, they were instructed to rate the pain experienced at four phases during the study. Endodontic access was performed five minutes after the second injection (buccal infiltration) and pain experience was compared between the three groups.

The results were interesting and showed large variations (clinically significant) between the three groups. Articaine was associated with a higher success rate (83.3\%) compared with lidocaine $(57.1 \%)$ and mepivacaine $(59.95 \%)$. Also, pain experience ratings were significantly lower in the articaine group compared with those in the lidocaine group and mepivacaine group.

With this in mind, it would seem that a supplemental buccal infiltration with articaine following IANB can be administered in teeth with irreversible pulpitis for maximum anaesthesia.

Aslam Alkadhimi

Specialist Registrar, Eastman Dental Institute UCL, London and Buckinghamshire Healthcare NHS Trust

\title{
Are your patients fit for radiation?
}

Presenting pre-radiotherapy dental status of head and neck cancer patients in the novel radiation era. Br Dent J 2020; 228: 435-440. https://doi. org/10.1038/s41415-020-1327-y

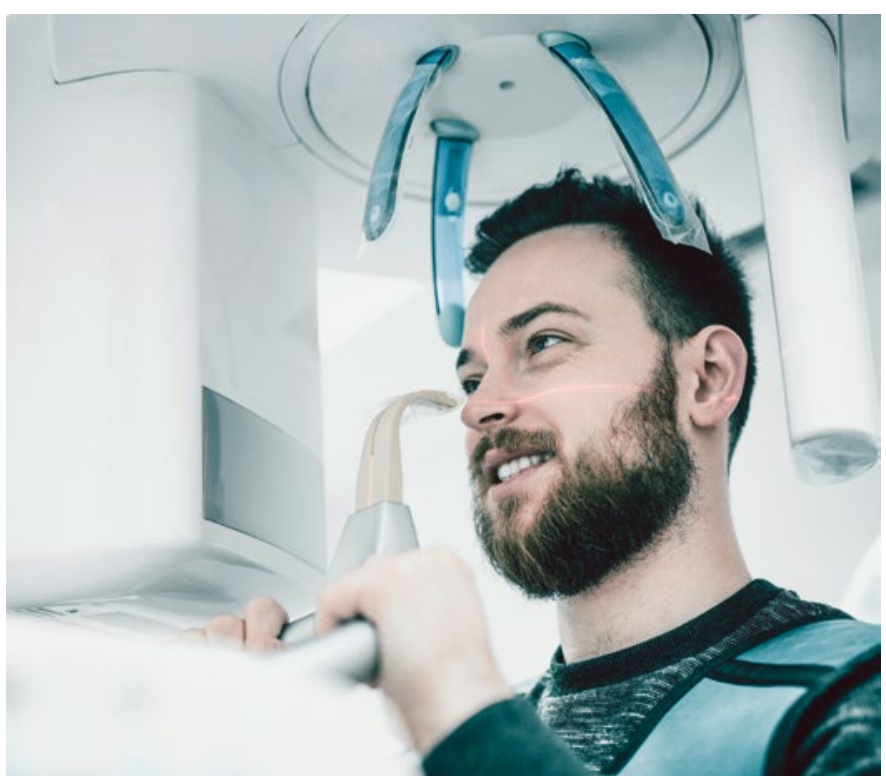

Head and neck cancer (HNC) is ranked the eighth most common cancer in the UK and accounts for approximately $3 \%$ of new cancer cases each year (12,000 cases). ${ }^{1}$ Till today, radiotherapy remains a primary tool to treat cancer. With the introduction of intensity-modulated radiotherapy treatment (IMRT), the radiation beam is limited to just the shape of the tumour, sparing the surrounding tissues from exposure. Consequently, reducing the chances of post-radiotherapy side effects. However, some have wondered if HNC patients have a poor dentition not because of the effects of radiotherapy, but because of their sub-standard oral health prior to radiotherapy.

The aim of this retrospective cohort study is to assess the preradiotherapy dental status of $886 \mathrm{HNC}$ patients with respect to patients factors (age, gender, smoking status) and tumour demographic (size, sub-site, nodal involvement). All patients included in this study were dentate and treated only by IMRT.
Firstly, there was a strong male predilection (72\%), with the most common age group being 55-64 years old (33\%). Oropharyngeal cancer was found to be the most frequent sub-site (36\%). This is of particular concern as tooth-bearing areas around the site of oropharyngeal cancer often receive a greater radiation dose compared to others. With high survival rates of oropharyngeal cancer, ${ }^{2}$ these teeth are often present in the mouth for longer after radiotherapy, putting them at an elevated risk of developing osteoradionecrosis.

Unsurprisingly, this study found smoking to be a significant factor in the development of HNC. Seventy-two percent of the cohort have a history of smoking, together with a higher DMFT index and greater horizontal bone loss compared to never-smokers. However, interestingly, the mean number of teeth present found in this cohort was only 19.8. Below the threshold of 21 teeth for a functional dentition and well below the mean number of teeth (25.7) amongst dentate adults according to the latest UK Adult Dental Survey. ${ }^{3}$ With this data, the authors highlight that HNC patients are well behind the general population in terms of oral health.

In summary, oropharyngeal cancer accounts for over one third of all HNC cases. But with a better prognosis ${ }^{2}$ there are now more survivors. The authors concluded that dental management of HNC patients needs to be revised, with a view to try and support a functional dentition while avoiding osteoradionecrosis and other post-radiotherapy adverse effects as long as possible.

\section{References}

1. Head and neck cancers statistics. Cancer Research UK. 2020. Available at https://www. cancerresearchuk.org/health-professional/cancer-statistics/statistics-by-cancer-type/ head-and-neck-cancers-heading-Zero (accessed April 2020).

2. Mehanna H, Evans M, Beasley M et al. Oropharyngeal cancer: United Kingdom National Multidisciplinary Guidelines. J Laryngol Otol 2016; 130: S90S96.

3. Adult Dental Health Survey 2009 - Summary report and thematic series - NHS Digital. NHS Digital. 2020. Available at https://digital.nhs.uk/data-and-information/publications/ statistical/adult-dental-health-survey/adult-dental-health-survey-2009-summary-reportand-thematic-series (accessed April 2020). 